\title{
Prevalence of antibodies against SARS-CoV-2 in hemodialysis patients
}

\author{
Giovanna Yazmin Arteaga-Müller ${ }^{1} \cdot$ Mara Olivo-Gutierrez $^{1} \cdot$ Karla Lizbeth Favela-Aragon $^{2}$. \\ Paola Alejandra Hernández-Castillo ${ }^{2}$. Victor Esquivel-Gomez ${ }^{1} \cdot$ Adrian Camacho-Ortiz $^{2,3}$ []
}

Received: 25 November 2020 / Accepted: 11 April 2021 / Published online: 23 April 2021

(C) The Author(s), under exclusive licence to Springer Nature B.V. 2021

Editor,

Elderly patients with comorbidities, such as diabetes and hypertension, are more susceptible to severe COVID-19. Hemodialysis (HD) patients have suboptimal immunity $[1,2]$ in addition to the expanded risk of being in constant contact with medical facilities. Contingency strategies focus on identifying patients with specific symptoms, using PCR-based testing [3]. There is little information on the use of rapid serological tests to demonstrate the presence or absence of antibodies [4]. This strategy is readily accessible to the medical staff without formal laboratory training.

All patients in our HD unit were tested within the 10 days after the detection of 1 confirmed case by positive PCR test, on May 21st, 2020. Samples ( $n=154)$ were obtained by pinprick using the COVID-19 IgG/IgM Rapid Test Cassette, (Healgen Scientific Limited Liability Company. Houston, USA), and these tests have sensitivity $90.11 \%$, specificity $98.46 \%$, and accuracy $95.31 \%$.

The mean age was 54.3 years $(19-87), 52.6 \%(n=81)$ were female, $61.7 \%$ attending twice a week, $32.9 \%$ thrice a week, and $2.7 \%$ once a week. The chronic kidney diseases (CKD) were: diabetic nephropathy $(58.3 \%)$, hypertension (18.8\%), glomerulopathies (4.7\%), and neurogenic bladder $(3.7 \%)$.

Out of the 154 test that were performed, 18 patients were positive for COVID-19, 11 tested positive for IgG (61.1\%\%),

Adrian Camacho-Ortiz

acamacho_md@yahoo.com

1 Department of Nephrology, Hospital Universitario "Dr. José Eleuterio González", Universidad Autónoma de Nuevo León, Monterrey, México

2 Department of Hospital Epidemiology, Hospital Universitario "Dr. José Eleuterio González", Universidad Autónoma de Nuevo León, Monterrey, México

3 Department of Infectious Diseases, Hospital Universitario "Dr. José Eleuterio González", Universidad Autónoma de Nuevo León, Monterrey, México
3 for IgG and $\operatorname{IgM}(16.7 \%)$, and 4 had positive IgM only (22.2\%) (Fig. 1), and 4 (22.2\%) of these patients shared a HD session with the patient positive PCR. Only 33\% of the antibody-positive patients presented at least 1 symptom with an average of 9.5 days before sample collection; predominantly cough, fever, dyspnea, and diarrhea, and $67 \%$ were pauci-symptomatic. Of the antibody-negative patients $(n=136), 81 \%(n=110)$ were pauci-symptomatic and the remaining 19\% $(n=26)$ referred at least 1 symptom in the last 5 days. Only the patient in the initial case had severe pneumonia, the rest of the patients with positive antibodies presented mild and moderate symptoms.

Antibody-positive patients had a mean age which was 52.6 years, $51 \%$ were female, the etiology of CKD diabetes $77.8 \%, 72.2 \%$ attended twice a week, and public transportations was used by $61.1 \%$.

A previous study demonstrated the high transmissibility of SARS-CoV-2 by the rapid appearance of COVID-19 among 230 dialysis patients with a total of $37 \mathrm{HD}$ patients and 4 members of the medical staff infected, post-performing tests after the first case [5]. In our center, 11 patients were identified with $\operatorname{IgG}$ antibodies, 3 with $\operatorname{IgG}$ and $\operatorname{IgM}$, and 4 only with IgM. With this information, a stratification of patients could be made in every hemodialysis unit.

Of the 18 antibody-positive patients, $33 \%$ had mild symptoms compatible with COVID-19 compared to $19 \%$ of the patients with negative antibodies. The term pauci-symptomatic was used for patients with CKD in HD treatment, since this population frequently presents symptoms associated with volume overload, such as dyspnea and fatigue, for that many patients with CKD curse unnoticed SARS-CoV-2 infection.

There was no difference in symptoms between antibody positive vs negative patients, suggesting that mild symptoms are not an adequate screening tool for COVID-19 in this population. We acknowledge limitations; mainly, the lack of FDA approved antibody testing that at the time of the study was not available in our region. 
Fig. 1 Flow chart illustrating study population selection
Flow chart illustrating study population selection

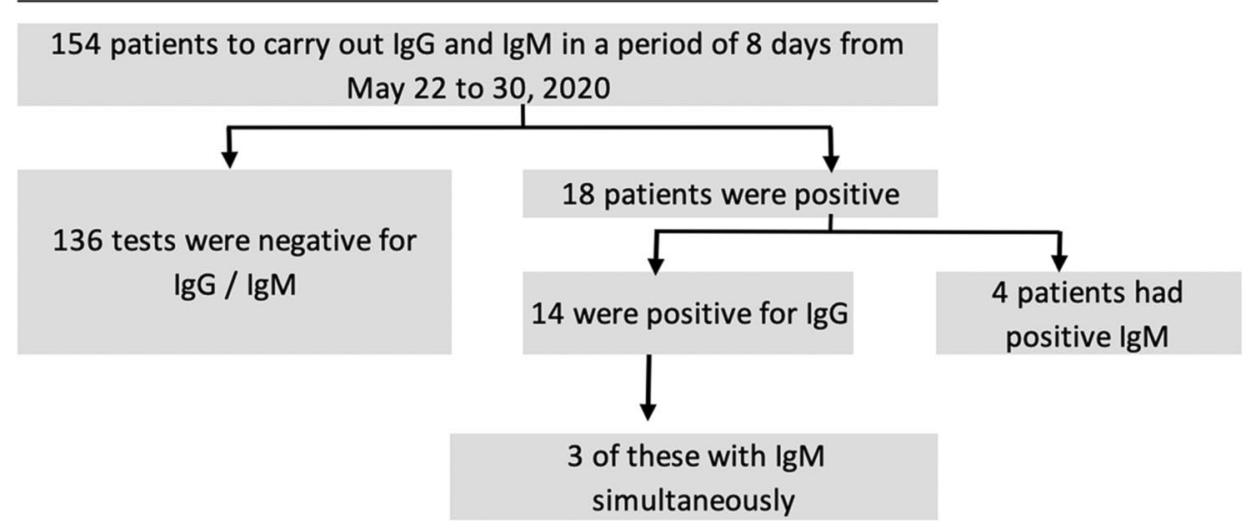

In the present study, the prevalence of positive antibodies against SARS-CoV-2 in HD patients was 27.36\%, 12 weeks after the first case was identified in the region. Determining the presence of antibodies could help to rapid diagnostic, attention, and severity classification in addition to stratify areas, personnel, and schedules for patients to avoid the rapid transmission.

\section{Declarations}

Conflict of interest The authors have declared that no conflict of interest exists.

Ethical approval All procedures performed were in accordance with the ethical standards research committee. Being an action carried out for early detection during the contingency of the COVID-19 pandemic, an approval number was not required. Informed consent was obtained from all individual participants included in the study.

\section{References}

1. Ishigami $\mathbf{J}$ et al (2019) Clinical epidemiology of infectious disease among patients with chronic kidney disease. ClinExpNephrol 23:437-447

2. Snigdha R, Chandrika C et al (2019) Vaccination in chronic kidney disease. Adv Chronic Kidney Dis 26(1):72-78

3. Xiang F, Wang X et al (2020) Antibody detection and dynamic characteristics in patients with COVID-19. Clin Infect Dis 71(8):1930-1934

4. Weinstein MC, Kenneth A, Freedberg KA et al (2020) Waiting for certainty on Covid-19 antibody tests at what cost? N Engl J Med 383(6):e37

5. Yiqiong M, Diao Bo B et al (2019) Novel coronavirus disease in hemodialysis patients: report from one HD center in Wuhan, China. MedRxiv. https://doi.org/10.1101/2020.02.24.200227201

Publisher's Note Springer Nature remains neutral with regard to jurisdictional claims in published maps and institutional affiliations. 